\title{
Bilder der Judenfeindschaft in den Werken von Jakob Wassermann
}

\author{
Leyla Coşan (iD), Istanbul - Habib Tekin (iD, Istanbul \\ https://dx.doi.org/10.37583/diyalog.958456
}

\begin{abstract}
Deutsch)
Der deutsch-jüdische Autor Jakob Wassermann (1873-1934) gehört zu den meistgelesenen und produktivsten Schriftstellern seiner Zeit. In seinem literarischen Schaffen sind autobiografische Züge und Themen über die Juden sowie das Judentum vorzufinden. Ziel dieses Beitrages soll es sein, die Bilder der Judenfeindschaft in seinen ausgewählten Werken, darunter Christian Wahnschaffe, Der Moloch, Der Fall Maurizius, Das Vorspiel: Sabbatai Zewi, Die Juden von Zirndorf und Die Geschichte der jungen Renate Fuchs, zu analysieren und zu zeigen, wie sehr die realhistorischen Gegebenheiten der stereotypen Topoi in der deutsch-jüdischen Literatur des 20. Jahrhunderts dargestellt werden. Das Konzept des »Übermenschen« im Sinne von Nietzsche sticht dann als der Ausweg für den Autor hervor, sodass die Auseinandersetzung mit dem »Anderen« ermöglicht und seine Leserschaft bezüglich der voreingestellten Feindschaft sensibilisiert werden kann. Dadurch kann die Möglichkeit eines Abbaus von Stereotypen durch Liebe und Gerechtigkeit erreicht werden, in dem sich die Menschen nämlich zur »Höherentwicklung« und der Hinwendung zum innersten Selbst bereit erklären.
\end{abstract}

Schlüsselwörter: Bilder der Judenfeindschaft, Jakob Wassermann, Stereotype, Assmann, Übermensch.

\section{Abstract (English)}

Images of Antisemitism in the works of Jakob Wassermann

Jakob Wassermann (1873-1934) is one of the most widely read and productive authors of his time. In his literary works, there are autobiographical elements and themes about the Jews and Judaism. The aim of this contribution is to analyse the images of antisemitism towards Jews in his selected works, such as Christian Wahnschaffe, Der Moloch, Der Fall Maurizius, Das Vorspiel: Sabbatai Zewi, Die Juden von Zirndorf and Die Geschichte der jungen Renate Fuchs, and to show how the real-historical conditions of the stereotypical topoi are represented in German-Jewish literature of the $20^{\text {th }}$ century. The concept of the »Übermensch" in the sense of Nietzsche then stands out as the way out for the author, so that the confrontation with the »other « is enabled and his readership is sensitised to the pre-set enmity. By doing so, the possibility to destruct stereotypes through love and justice can be made possible, if men declare that they are ready for the »higher development« and the devotion to the innermost self.

Keywords: Images of Antisemitism, Jakob Wassermann, Stereotype, Assmann, Übermensch. 


\section{EXTENDED ABSTRACT}

Jakob Wassermann (1873-1934) is one of the most widely read and productive authors of his time. In his literary works, there are autobiographical elements and themes about the Jews and Judaism. The aim of this contribution is to analyse the images of antisemitism towards Jews in his selected works, such as Christian Wahnschaffe (1919), Der Moloch (1902), Der Fall Maurizius (1928), Das Vorspiel: Sabbatai Zewi (1925), Die Juden von Zirndorf (1897) and Die Geschichte der jungen Renate Fuchs (1900), and to show how the real-historical conditions of the stereotypical topoi are represented in German-Jewish literature of the $20^{\text {th }}$ century. The concept of the $»$ Übermensch $«$ in the sense of Nietzsche then stands out as the way out for the author, so that the confrontation with the »other « is enabled and his readership is sensitised to the pre-set enmity. By doing so, the possibility to destruct stereotypes through love and justice can be made possible, if men declare that they are ready for the »higher development « and the devotion to the innermost self.

The feeling of animosity towards the Jews and Judaism is sui generis a socially, historically, culturally and collectively based resentment, which, starting from its origin, spreads and manifests itself throughout Europe over the centuries. In the course of time, certain images emerge that preserve and further force the feeling of enmity. These mostly stereotypical images are passed on to the next generations and internalised as such. In the $15^{\text {th }}$ century of Europe, anti-Semitism also reached one of its climaxes. At that time, inquisition tribunals were established in Spain and Jews were forcibly converted, baptised, murdered and expelled on European soil. In this sense, the widespread anti-Semitism can be traced back to the existence of anti-Jewish images that have been passed on from one generation to the next over millennia.

Therefore, in the theoretical part, the reasons for the emergence of the antiJewish images are presented. The stereotypes that result from the perception of the "other" and the "unknown" leads to a certain pre-attitude towards specific ethnic groups - in this case the Jewish one - and ensure a separate perception of foreignness. History, and therefore also historical facticity, plays a relevant role. In this regard and in addition to the image of the other and the self-image, the reasons for hostility towards Jews as a collective fear of the West are outlined.

In the selected works, anti-Jewish images are used to a far greater extent. For example, apart from the character Agathon in Die Juden von Zirndorf, all the other protagonists in the works to be analysed are mostly of non-Jewish descent and act as carriers of ideas and/or messages, on the basis of which Wassermann projects his idea of the perfect human being, the conception of the »Übermensch« and justice.

By illustrating the collective fears of the West, depicting the images of hostility towards Jews, and thus the confrontation of one's own with the »other«, Wassermann instils love, respect, good-naturedness, and humanity to his readers. So, the aim is to break down the prejudices that have been handed down over the centuries and encourage people to do better with regard to the »other«. 
The solution in form of the conception of the »Übermensch« wants every last man to turn to a higher human-being, furthermore to evolve love, understanding and respect for his fellows and, in this way, to absorb and obliterate the negative within.

All in all, the aim of the present work is to prove and analyse stereotypical images of hostility towards Jews in the works of Jakob Wassermann. In particular, it is about stereotypical images that can be proven to have a cognitive anchoring and openly show the transmission of hostility towards Jews. 


\section{Einleitung}

Das Gefühl der Animosität gegenüber den Juden und dem Judentum ist sui generis ein sozial, historisch, kulturell und kollektiv fundiertes Ressentiment, das sich von seinem Ursprung ausgehend über die Jahrhunderte in ganz Europa verbreitet und manifestiert. Dabei entstehen im Laufe der Zeit bestimmte Bilder, die das Gefühl der Feindschaft konservieren und weiter forcieren. Diese zumeist stereotypen Bilder werden an die folgenden Generationen weitergegeben und als solche verinnerlicht. Im Europa des 15. Jahrhunderts erreicht der Antisemitismus auch einen seiner Höhepunkte. Zu jener Zeit werden in Spanien Inquisitionstribunale errichtet und Juden auf europäischem Boden zwangskonvertiert, getauft, ermordet und vertrieben. Der weitverbreitete Antisemitismus lässt sich in diesem Sinne auf die Existenz judenfeindlicher Bilder zurückführen, die unhinterfragt über Jahrtausende von einer Generation in die nächste tradiert werden.

Es geht demgemäß in dem vorliegenden Aufsatz ${ }^{1}$ um die Darstellung der realhistorischen Gegebenheiten stereotyper Bilder in der deutschen Literatur des 20. Jahrhunderts. Hierbei wird der Versuch unternommen, die Tradierung der Bilder der Judenfeindschaft anhand ausgewählter Werke Jakob Wassermanns ${ }^{2}$ nachzuzeichnen. In der Analyse werden die Werke (1) Christian Wahnschaffe, (2) Der Moloch, (3) Der Fall Maurizius, (4) Das Vorspiel: Sabbatai Zewi, (5) Die Juden von Zirndorf und (6) Die Geschichte der jungen Renate Fuchs herangezogen.

Deshalb werden im theoretischen Teil die Gründe für die Entstehung der judenfeindlichen Bilder ausfindig gemacht. Die Stereotype, die durch die Wahrnehmung des Anderen und Fremden erfolgen, resultieren in einer bestimmten Voreinstellung gegenüber gezielten Ethnien - im vorliegenden Fall der jüdischen - und sorgen für eine abgesonderte Fremdheitswahrnehmung. Die Geschichte - und deshalb auch die historische Faktizität - spielt dabei eine relevante Rolle. Diesbezüglich werden neben den Fremd- und Selbstbildern auch die Gründe der Judenfeindschaft als kollektive Angst des Abendlandes skizziert. ${ }^{3}$

\footnotetext{
${ }^{1}$ Der vorliegende Artikel ist eine thematische Einführung in meine Dissertation, die 2021 eingereicht wurde.

${ }^{2}$ Für die Autobiografie und weitere Reden und Schriften vgl.: Wassermann, Jakob (1984b): Mein Weg als Deutscher und Jude. In: Rodewald, Dirk (Hrsg.) (1984): Jakob Wassermann. Deutscher und Jude. Reden und Schriften 1904-1933. Heidelberg: Lambert Scheider. S. 35-131. Für weitere Schriften vgl.: Rodewald, Dirk (Hrsg.) (1984): Jakob Wassermann. Deutscher und Jude. Reden und Schriften 19041933. Heidelberg: Lambert Scheider. Für die Biografie der ersten Ehefrau vgl:: Wassermann-Speyer, Julie (1923): Jakob Wassermann und sein Werk. Wien und Leipzig: Deutsch-österreichischer Verlag. Für die Biografie der zweiten Ehefrau Marta Karlweis vgl:: Karlweis, Marta (1935): Jakob Wassermann, Bild, Kampf und Werk. Mit einem Geleitwort von Thomas Mann. Amsterdam: Querido. Für die empfohlene Biografie vgl.: Kraft, Thomas (2008): Jakob Wassermann. München: DTV.

${ }^{3}$ Für die Einführung in Fremdheitswahrnehmungen, Fremd- und Selbstbilder, und die Entstehung von Stereotypen siehe: Lüsebrink, Hans-Jürgen (2012): Interkulturelle Kommunikation. Interaktion, Fremdwahrnehmung, Kulturtransfer. 3. aktualisierte und erweiterte Auflage. Stuttgart/Weimar: Metzler; Bitterli, Urs (2004): Die 'Wilden' und die 'Zivilisierten': Grundzüge einer Geistes- und Kulturgeschichte der europäischen-überseeischen Begegnung. München: Beck; Quasthoff, Uta M. (1973): Soziales Vorurteil und Kommunikation - Eine sprachwissenschaftiche Analyse des Stereotyps. Ein interdisziplinärer Versuch im Bereich der Linguistik, Sozialwissenschaft und Psychologie. Frankfurt
} 
In den ausgewählten Werken werden antijüdische Bilder in sehr großem Umfang eingesetzt. Beispielsweise sind außer der Figur Agathon in Die Juden von Zirndorf alle anderen Protagonisten in den $\mathrm{zu}$ analysierenden Werken meist nichtjüdischer Abstammung und fungieren als Ideenträger, anhand derer Wassermann seine Vorstellung vom vollkommenen Menschen, dem »Übermenschen«, und seine Auffassung von Gerechtigkeit projiziert.

\section{Fremdheitswahrnehmung, Stereotyp, Vorurteil}

Die Bilder der Judenfeindschaft entstehen aufgrund unterschiedlicher Fremdheitswahrnehmungen des Einen gegenüber dem Anderen, d. h. in etwa, wie eine Kultur die andere und meist fremde Kultur wahrnimmt. Dabei gibt es Selbst- und Fremdbilder. Die Selbstbilder werden als sogenannte »Images« bezeichnet, die eine Person oder eine Gruppe von Menschen von sich preisgibt. Sie sind eine Vorstellung oder ein Bild, das ein Einzelner oder Kollektiv von einer anderen Person, Gruppe oder Sache hat. In dieser Hinsicht sind sowohl Selbst- als auch Fremdbilder subjektiv und beschäftigen sich primär mit dem Anderen. Die Definition vom Image lässt sich demnach folgendermaßen festhalten:

Das Image ist verallgemeinert das Erscheinungsbild von einem bestimmten Phänomen, einem Ereignis oder einer Region. [...]. Das Image ist das Ergebnis einer Vielzahl von partiellen Vorstellungsbildern, die in ihrer Zusammensetzung sehr unterschiedlich sein können. Je stärker die jeweilige Sichtweise von objektiven Faktoren beeinflußt ist, um so einheitlicher, gefestigter und realitätsnäher ist das Image des betreffenden Sachverhalts. Umgekehrt läßt eine Dominanz subjektiver Faktoren eher ein realitätsfernes Image erwarten. [...]. Sie entstehen zum einen durch den direkten Kontakt mit der Umwelt oder dem jeweiligen Sachverhalt. Die Inhalte können zum anderen auch nur passiv durch die Medien vermittelt werden. Als weitere Komponenten sind schließlich die individuellen Wahrnehmungen, Gefühle, Vorstellungen und Erfahrungen des jeweiligen Images von entscheidender Bedeutung.

am Main: Athenäum Fischer; Bausinger, Hermann (1988): Stereotypie und Wirklichkeit. In: Jahrbuch Deutsch als Fremdsprache 14. S. 157-170. Für den kulturgeschichtlichen Ansatz der Begründung der Angst des Abendlandes siehe: Delumeau, Jean (1985): Angst im Abendland. Die Geschichte kollektiver Ängste im Europa des 14. bis 18. Jahrhunderts. 2 Bände. Hrsg. v. Johannes Beck. u. a. und übersetzt von Monika Hübner, Gabriel Konder und Martina Roters-Burck. Hamburg: Rowohlt. Für die Tradierung, Rekonstruktion und Vergegenwärtigung von Erinnerungen siehe: Assmann, Aleida (1994): Tradition, Evolution, Erinnerung. Überlegungen zum Strukturwandel kultureller Überlieferung. In: GoodmannThau, Eveline und Schmied-Kowarzik, Wolfdietrich (Hrsg.) (1994): Messianismus zwischen Mythos und Macht. Jüdisches Denken in der europäischen Geistesgeschichte. Berlin: Akademie. S. 89-199; Assmann, Aleida (1999): Erinnerungsräume, Formen und Wandlungen des kulturellen Gedächtnisses. München: Beck; Assmann, Jan (2000): Das kulturelle Gedächtnis. Schrift, Erinnerung und politische Identität in frühen Hochkulturen. München: Beck; Assmann, Jan (2017): Religion und kulturelles Gedächtnis. München: Beck; Assmann, Jan und Tonio, Hölscher (1988): Kultur und Gedächtnis. Frankfurt am Main: Suhrkamp. Und letztlich für die Bilder der Judenfeindschaft siehe: Rohrbacher, Stefan (1990): Eine Kreuztagung des 16. Jahrhunderts und ihr ikonographischer Kontext. In: Neusser Jahrbuch für Kunst, Kulturgeschichte und Heimatkunde. 35. Jg. S. 15-24; Rohrbacher, Stefan und Schmidt, Michael (1991): Judenbilder, Kulturgeschichte antijüdischer Mythen und antisemitischer Vorurteile. Hamburg: Rowohlt; Schoeps, Julius H. und Schlör, Joachim (Hrsg.) (1999): Bilder der Judenfeindschaft. Antisemitismus, Vorurteile und Mythen. Augsburg: Bechtermünz. 
Es kann auch vorkommen, dass Erscheinungsbilder aus dieser Perspektive von fremden Kulturen mit Stereotypen und Vorurteilen beladen wahrgenommen werden können, wie es der Fall bei der jüdischen Ethnie ist. Auf der einen Seite geben die Juden beispielsweise ein bestimmtes und meist in sich geschlossenes Selbstbild von sich ab und auf der anderen Seite werden sie von dem Anderen als fremd bezeichnet. Die mit Stereotypen versehene Einstellung gegenüber dem Anderen sorgt für eine Abgrenzung, die meist mit Angst und Unwissenheit zu begründen ist und sich in den kollektiven Ängsten einer Gemeinschaft, in diesem Falle der Angst des Abendlandes, ausdrückt. Die kollektiven Erscheinungen haben sich in Form der Panik und Aufstände, Verfolgung, Hexenverbrennung, Sündenbocktitulierung gezeigt. Die Aussagen und Lehren der Kirche waren ausschlaggebend. Die Sündenböcke sind laut kirchlicher Obrigkeit Ketzer, Hexen, Türken, Juden und dergleichen. Laut Charles Henry Lea (1911: 19), „[fällt] [d]as Vorurteil gegen die Juden, das während des Mittelalters bei weitem nicht überall bestand, in der Hauptsache, wenn nicht ganz, der Kirche zur Last.“ Die Kirche ist also nach Lea dafür zuständig, Feindbilder zu erstellen, um die eigene Machtposition gegenüber dem einfachen unmündigen Volk zu sichern.

Die jüdische Bevölkerung steht demnach als bestes Exempel für die Beschuldigung als »Brunnenvergifter« zur Verfügung. Juden wurden bereits vor der Pest und den Flagellanten beschuldigt, 1321 die Brunnen vergiftet zu haben (vgl. Delumeau 1985: 185). Deshalb wurden 1348 in Stuttgart Juden verbrannt, obwohl die Pest erst 1350 dort ausbrach. Dass auch Juden aufgrund der Krankheit starben, wurde ausgeblendet. Dahinter liegt die Idee, dass nach christlicher Sicht die Juden mittels der Brunnenvergiftung beabsichtigt haben sollen, die Christen gänzlich auszurotten. Die Judenfeindschaft rückt folglich in den Vordergrund. Sie sind die ausgewählten Sündenböcke in Krisenzeiten (vgl. Delumeau 1985: 413). Auch Schoeps erwähnt das Mitwirken der kirchlichen Obrigkeit, die er für die Entstehung der Stereotype und somit der Absonderung der Juden verantwortlich macht:

\footnotetext{
Das Dogma, die Juden seien von Gott verflucht und verworfen worden, weil sie Jesus nicht als Messias anerkannt und seine Kreuzigung veranlasst haben, dieses Dogma hat das christliche Judenbild durch die Jahrhunderte bestimmt [...]. Nicht die soziale Wirklichkeit, sondern die Anschuldigungen der Kirchenväter schufen das Stereotyp, das von Generation zu Generation weitergegebene Zerrbild des Juden. Als Urtypus des Sünders und Frevlers [...] wirkt der Jude als abschreckendes Beispiel. (Schoeps 1986: 105)
}

Die Bilder der Judenfeindschaft sind also nach Schoeps in erster Linie nicht die »Brunnenvergifter«, sondern, in Anlehnung an den »Gottesmörder«, die »Ritualmörder«, der »Antichrist«, »Wucherer«, »Hausierer«, »Schacherer«, »Kornjude«, »orientaler Jude«, »ewiger Jude«, »Ostjude« u. v. a.

\section{Bilder der Judenfeindschaft und ihre Tradierung}

In diesem Abschnitt werden Judenbilder dargestellt, die Wassermann in seinen Werken verwendet hat. Es handelt sich hierbei um die Verbreitung der negativ semantischkonnotierten antijüdischen Bilder. Sie werden demgemäß vorgeführt als »Gottes-« und 
»Ritualmörder«, »ewige Juden«, »Wucherer«, »Hausierer«, »Verräter«, »Antichristen«, »Ostjuden« u. v. a. (vgl. Hortzitz 1999). ${ }^{4}$

Es stellt sich die leitmotivische Frage, wie die Erinnerungen an die Bilder der Judenfeindschaft von einer Generation in die nächste weitergegeben und somit stereotype Topoi vergegenwärtigt und aufrechterhalten werden. Die Theorie der Tradierung von Erinnerung(en) und die Rekonstruktion werden anlehnend an die Forschungen von Aleida Assmann und Jan Assmann beantwortet.

Die These der Assmanns baut auf die von Maurice Halbwachs auf, und sie führen diese weiter aus. Darüber hinaus rücken sie den Begriff des kollektiven und kulturellen Gedächtnisses in den Mittelpunkt, welcher für die vorliegende Arbeit grundlegend ist.

Das Gedächtnis ist nach Assmann ein soziales Konstrukt und entsteht erst im Prozess der Sozialisation. Mit anderen Worten entwickelt ein Individuum sein eigenes Gedächtnis. Da im Laufe der Sozialisation, das kollektive Gedächtnis den Einzelnen besonders prägt, ist das individuelle Gedächtnis vom Kollektiv, in der er sich befindet, abhängig; deshalb auch die Bezeichnung vom kollektiven Gedächtnis. Kollektive haben zwar kein eigenes Gedächtnis, doch Einzelne der sozialen Gruppe mit einem individuellen Gedächtnis prägen das kollektive. Eine Vorbedingung hierzu wäre die Kommunikation und Interaktion innerhalb eines Rahmens, denn ,[w]ir erinnern nicht nur, was wir von anderen erfahren, sondern auch, was uns andere erzählen und was uns anderen als bedeutsam bestätigt und zurückgespiegelt wird [,] [...] [denn], es gibt keine Erinnerung ohne Wahrnehmung،“ (ebd.: 364). Die Zweckmäßigkeit dieser Theorie liegt darin, dass die Theorie des Erinnerns den Akt des Vergessens erklärt, da Einzelne sich selektiv an Dinge erinnern, die innerhalb eines Bezugsrahmens zur jeweiligen Gegenwart stehen. Diejenigen Elemente, an die man sich nicht zu erinnern vermag, stehen nicht in einem Bezugsrahmen und werden deshalb nicht vergegenwärtigt.

Das kulturelle Gedächtnis hingegen befasst sich mit Fixpunkten der Vergangenheit. Sie interessiert sich für die erinnerte Geschichte, nicht für die faktische Historizität. Vergangenheit schlägt sich nieder in symbolischen Figuren. Solche Erinnerungsfiguren lassen sich besonders im Judentum ausfindig machen wie zum Beispiel die Exodus-Überlieferung, die Vätergeschichten, die Landnahme oder das Exil. Nach Assmann kann man sagen, dass

\begin{abstract}
im kulturellen Gedächtnis faktische Geschichte in erinnerte und damit in Mythos transformiert wird. Mythos ist eine fundierende Geschichte, eine Geschichte, die erzählt wird, um eine Gegenwart vom Ursprung her zu erhellen. Der Exodus ist, völlig unabhängig von der Frage seiner Historizität, der Gründungsmythos Israels: als solcher wird er im Pessach-Fest begangen und als solcher gehört er ins kulturelle Gedächtnis des Volkes. Durch Erinnerung wird Geschichte zum Mythos. (Assmann 2000: 52)
\end{abstract}

\footnotetext{
${ }^{4}$ Auf die Entstehungsgründe der Bilder der Judenfeindschaft wird nicht näher eingegangen, da es den Rahmen der Arbeit sprengen würde.
} 
Wichtige Stellen der Assmann'schen Forschung zeigen dann die Merkmale des Erinnerns, die Erinnerungs- und Schriftkultur und Riten und Feste als identitätsstiftende Faktoren.

\section{Zu Christian Wahnschaffe}

Christian Wahnschaffe ist ein zweibändiger Roman, den Jakob Wassermann 1919 bei Samuel Fischer in Berlin publizierte. Thematisiert wird in dem Roman das Leben des gleichnamigen Protagonisten, die verschiedenen Stadien seiner persönlichen Entwicklung sowie sein Mensch- und Weltbild. Der wohlhabende österreichische Bourgeois und Sohn des reichen, protestantischen Großindustriellen steht mit seinem Wohlstandsleben und seiner Oberflächlichkeit im Vordergrund. Im Laufe der Zeit entsagt er dem Erbe seiner Familie und führt ein ärmlicheres Leben als zuvor.

In Christian Wahnschaffe werden auch zahlreiche Bilder der Judenfeindschaft und die vorurteilhafte Einstellung gegenüber den Juden dargestellt. Beispielsweise wird durch den Freund Christians, Felix Imhof, auf die Wurzellosigkeit der Juden aufmerksam macht. Darüber hinaus wird die Familie Wahnschaffe vorgestellt, wodurch ein Einblick in eine nicht-jüdische Familie gewährleistet ist. Durch diesen Blick werden typische Grundhaltungen gegenüber den Juden reflektiert und antijüdische Bilder wie etwa »Brunnenvergifter«, »Vampire«, »Blutsauger« und »Volksbetrüger« von Christians Vater vorgeführt:

\footnotetext{
Unsre Volksbetrüger füttern ihren Anhang mit dem frechen Märchen, als seien wir die Vampire, die das Blut der Unterdrückten trinken. Sie wissen nichts, die Brunnenvergifter, oder wollen nichts wissen von den Erschütterungen, Leiden und Entbehrungen, an die der friedliche Lohnsklave mit keiner Ahnung hinreicht. (Wassermann 2016a: 162)
}

Es wird sogar auf die eschatologische Hoffnung der Juden und die Erwartung auf einen Messias eingegangen. Besonders auffallend ist auch die Verwendung von zwei bestimmten Stellen aus der Bibel, zum einen Das Buch Jesaja und zum anderen Die Johannesoffenbarung. Letztere geht nicht nur auf die apokalyptische Zeit und die Schreckensherrschaft ein, sondern auch auf das Tier aus dem Meer, welches der Autor mit dem Ungeheuer symbolhaft darstellt (s. Wassermann 2016a: 194f.). Ferner tritt die Erzählung vom Propheten an mehreren Stellen auf: an den Wänden Christians und in der Bibelrezitation des »Judenhassers« (vgl. ebd.: 485) Amadeus. Somit wird die Einbettung zahlreicher jüdischer Topoi bestätigt. Durch die Familie Hofmann wird nämlich das Stereotyp des »Ostjuden« und des »Hausierers«, durch Eva Sorel und dem Diamanten die jüdische Mystik, das Motiv des »Wanderns« (darunter auch das »Ahasver-Motiv«), des »Lebensmittelschachers" und »Kornjudenhändlers«, und die »jüdische Tarnung«, und durch (vgl. ebd.: 133, 184, 217, 219, 231, 265, 284f.) Johanna das jüdische Stereotyp der »ikonographischen Tradition der Darstellung von Häßlichkeit« (vgl. ebd.: 265, 430). Weitere im Werk vorhandene Stereotype sind das Judenzeichen vom »gelben Fleck« (vgl. Wassermann 2016a: 312f.) an der Kleidung und das »Opfermotiv«, welches durch den Vergleich mit der Tochter des Jephta aus der 
Bibel rekonstruiert wird. (vgl. Wassermann 2016a: 475, 485). Darüber hinaus wird durch die »heilige« Ruth die Charakteristik des selbstlosen Übermenschen, durch Michael die Möglichkeit des Abbaus von Stereotypen, unter der Bedingung der Willensbereitschaft, und durch die Liebe zwischen den Antisemiten Voß und der Jüdin Johanna die Vorstellung und Möglichkeit von vorurteilsfreier Beziehung ermöglicht.

Die Einblicke in jüdische Welten und typische Judenschicksale werden anhand der erwähnten Figuren aufgeführt. Der Leitfaden für die geschilderten Erzählungen ist dabei der neutral ausgestattete Ideenträger und Protagonist Christian Wahnschaffe, der zwar aus einer judenintoleranten Familie stammt, jedoch aufgrund seiner Natur ein konfessionstolerantes Selbstbild von sich gibt.

\section{Zu Der Moloch}

Der Moloch ist das zweite Werk von Jakob Wassermann mit ausgiebig jüdischem Inhalt und thematisiert die Entführung eines jüdischen Mädchens und die Suche nach ihr. Hier werden erneut Judenschicksale anhand der Vorbilder in Europa, so etwa der Spanischen Inquisition, nachgezeichnet und vergegenwärtigt. Durch die Verwendung von stereotypen Bildern werden das elende Schicksal des Judenmädchens und das Verbrechen an einer jüdischen Familie aufgezeigt. Die abwesende Justiz, das NichtsTun und Nichts-Bewirken-Können bilden dabei leitmotivische Aspekte im ganzen Roman, mit dem der Protagonist sich hoffnungsvoll jedoch wirkungslos auseinandersetzt. Es stellt sich heraus, dass die zunehmende Anzahl der Verwendung von jüdischen Stereotypen wie etwa »Kindesopfer«, »Gottes- und Ritualmörder«, »Hausierhandel«, »Schacher«, »Wucher«, »Blutsaugermotiv«, die »zweitausendjährige Schuld « und das »jüdische Gesindel« die tiefgründige Auseinandersetzung mit stereotypen Bildern darstellt:

\footnotetext{
$\mathrm{Na}$, die Juden seien eben keine rechten Männer, sie behexten das Vieh und zu Ostern schlachten sie Christenkinder. [...] Der Jud ist arm, hat neun Kinder zu Haus und wenn er zu Gericht geht, wird er auch sein Recht bekommen. (Wassermann 2017a: 13)
}

Allein der Titel des Werkes »Moloch« trägt eine Doppelbedeutung. Zum einen wird er als eine Kritik an die Großstadt gedeutet, zum anderen aber auch als ein Akt der Opfergabe oder sogar die Gottheit selber, die das Opfer empfängt. Ganz grundlegend ist im Roman die Ungerechtigkeit, m. a. W., die abwesende Justiz für Juden und die Suizidproblematik. Aufgrund der Ungerechtigkeit hat nämlich der Protagonist sich das Leben genommen und kurz zuvor akzentuiert, dass ein Leben ohne Gerechtigkeit nicht möglich sei. Dadurch ergibt sich, dass Wassermann mit der Verwendung der Stereotype und des Problems der Gerechtigkeit, die er als ein von Natur aus gegebenes Recht sieht, auf die unmenschliche Handlung aufmerksam macht. Die Hoffnungslosigkeit wird durch verschiedene Stadien der Handlungen von Juden und gerechtigkeitssuchenden Figuren im Roman zugespitzt nachgezeichnet und die Tatsache der abwesenden Justiz in ihrer Bloßheit zur Schau gestellt:

,Sehn Sie nur an', fuhr die magere Jüdin fort und hob sibyllenhaft den Kopf, ,wie es bestellt ist mit dem Recht. Für die armen Leute gibt's kein Recht, für arme Juden gibt's 
gar kein Recht. Und mit was kann ich dienen? Mit wem hab ich das Vergnügen?

(Wassermann 2017a: 23)

Die diskursinternen Figuren haben nämlich beim Bezirksrichter, Staatsanwalt, Ministerpräsidenten, Justizminister und Minister für Galizien nach Gerechtigkeit angefragt, jedoch hat jedes Mal die Macht an »den Mauern des Klosters« ein Ende.

\title{
Zu Der Fall Maurizius
}

Im Werk Der Fall Maurizius geht es primär um die Figur Warschauer-Waremme und Etzel Andergast, dem Protagonisten. Ersterer führt sich als ein aufgrund von strategischen Gründen tarnender Jude vor - wie Heine auch - und letzterer fungiert ebenfalls wie die vorherigen Protagonisten als ein Ideenträger. Folglich gerät Etzel Andergast aufgrund der an Maurizius verübten Ungerechtigkeit und des Justizmordes in eine psychisch instabile Lage. Die Abwertung des jüdischen Schicksals sowohl im privaten als auch im öffentlichen Leben und der im Roman vorhandene Europagedanke werden von Waremme-Warschauer mit weiteren stereotypen Bildern verstärkt zum Ausdruck gebracht:

\begin{abstract}
Europa den Rücken kehren heißt noch nicht, ohne Europa existieren können. Er fing an zu verstehen, was Europa für einen Menschen wie ihn eigentlich war. Nicht bloß seine persönliche Vergangenheit, sondern die Vergangenheit von dreihundert Millionen Menschen. Zugleich das, was er davon wußte, was er davon im Blute hatte. [...] Europa war nicht bloß die Summe der Bindungen in seiner individuellen Existenz, Freundschaft und Liebe, Haß und Unglück, Gelingen und Enttäuschung, es war, ehrwürdig und unfaßbar, die Existenz eines Ganzen seit zweitausend Jahren, Perikles und Nostradamus, Theoderich und Voltaire, Ovid und Erasmus, Archimedes und Gauß, Calderon und Dürer, Phidias und Mozart, Petrarka und Napoleon, Galilei und Nietzsche. (Wassermann 2016b: 222)
\end{abstract}

Das Problem der Gerechtigkeit rückt hier besonders in den Vordergrund und erlebt seinen Höhepunkt innerhalb der Wassermann'schen Werke, denn es findet ein Justizmord statt. Mit dem Protagonisten, dem der Autor ,,jüdisches Blut in den Adern“ (Wassermann 2016b: 70) zuschreibt, und Waremme-Warschauer werden die jüdische Identitätsproblematik und Gerechtigkeit erneut ins Zentrum gerückt. Wie zu erkennen ist, bringt der Autor das natürliche und angeborene »Urrecht« der Gerechtigkeit explizit zum Ausdruck. Für die Gerechtigkeitsproblematik verwendet Wassermann WaremmeWarschauer, der seine Identität aus strategischen Gründen leugnet.

Die Täuschung des Mordes durch Waremmes Meineid und die Verlagerung der Schuld auf Maurizius deuten auf die Konzentration der Macht bei der jüdischen Figur. Hiermit wird nicht nur der »passive $\aleph^{5}$ und »leidende« Jude dargestellt, sondern ebenso auch der mächtige, der, als die einzige Figur im Roman, die Handlung vorantreibt und

\footnotetext{
5 An dieser Stelle wird im Gegensatz zu Bubers »Muskeljude« das stereotype Bild des passiven Ghettojuden dargestellt. Der zionistische Stereotyp des maskulinen »Muskeljuden« steht im Kontrast zu den passiven, effeminierten und widerstandlosen Ghettojuden (s. hierzu Buber, Martin (1960): Jüdische Renaissance. In: Buber, Martin (1960): Die Jüdische Bewegung, Band 1. Auflage 2. Berlin: Jüdischer Verlag. S. 7-16, hier: S. 9).
} 
die Ungerechtigkeit lösen kann. Mit seinem schauspielerisch-betrügerischen Auftreten im Gerichtssaal und seine „sublimierte Verwandlungskunst“ (Wassermann 2016b: 212) der sofortigen Anpassung an die jeweiligen Umstände, hält er das Schicksal des Opfers in den Händen. Dadurch wird kenntlich gemacht, dass Wassermann hier seine jüdische Figur mit den Stereotypen der »Dunkelmänner«, »Verräter« und »Betrüger«, wie etwa dem des verräterischen Apostels Judas Ischariot, ausstattet.

[Waremme] ist ein Judas Ischariot und Ahasver, der durch entfernte Völker und Länder wandert (Amerika!), mit seiner einzigen Hoffnung im Osten, wo eine Tochter, die er nie gesehen hat, auf ihn wartet und wo er schließlich Ruhe für sein müdes Haupt und Trost für seine sturmzerzauste Seele finden wird. (Shaked 1986: 109)

Waremmes Missbilligung über die Haltung seiner Stammesgenossen, die sich wie „Sklaven der Spartaner“ (Wassermann 2016b: 206) verhalten, und die Abwertung über die „listige Erfindung“ (ebd.: 205) der jüdischen Haskala werden ebenfalls zum Ausdruck gebracht. Die Vertrieben- und Ausgeschlossenheit haben den reformatorischen Geist Waremmes, vergleichbar etwa mit einem ,jüdischen Führer“ (ebd.: 206), „einem Luther des Judentums“ (ebd.), hervorgerufen. Weitere im Roman verwendete Stereotype sind »Brunnenvergifter«, »Ritualmörder« und die »blutgierige« und »unbarmherzige Wucherin«:

[V]ielleicht ist Ihnen [...] zu Ohren gekommen, was sich gegenwärtig hierzulande abspielt? [...] . . . na, lassen Sie mich nur ein Beispiel anführen, als Grabsteine in jüdischen Friedhöfen zu demolieren, meinen Sie nicht? Was sagen Sie dazu, geschätzter Mohl? Grabsteine demolieren . . . he? Friedhöfe schänden . . . Das ist neu in der Kulturgeschichte, he? Dernier cri. Ich finde, daß dagegen alle Brunnenvergiftungs- und Ritualmordexzesse zwar blutrünstige und hirnlose, aber, wenn man großzügig denkt, durch Wahn und Leidenschaft entschuldbare Veranstaltungen waren, was finden Sie? Sie schweigen, kleiner Mohl? (Wassermann 2016b: 205)

Darüber hinaus werden die ,uralten Märchenerfindungen“ gezeigt und weitere biblische Erzählungen wie etwa die Geschichten von Saul und König Salomon erwähnt. Diese hier vorgeführten Stereotype sind ein fester Bestandteil des kollektiven Gedächtnisses und existieren über Jahrhunderte. An diese wird erinnert und somit ein Mythos erzeugt, der im Sinne der Theorie von Assmann die Vergegenwärtigung fundierter Geschichte darstellt. Die Existenz dessen im kollektiven Gedächtnis ist also ein Indiz für die Absonderung oder Voreingenommenheit gegenüber den Juden.

\section{Zu der Agathon-Handlung - Das Vorspiel: Sabbatai Zewi, Die Juden von Zirndorf und Die Geschichte der jungen Renate Fuchs}

Der Roman Die Juden von Zirndorf nimmt den Großteil des jüdischen Sachgehalts innerhalb der Wassermann'schen Werke auf, denn im Vorspiel werden die Judenhoffnung und die Erwartungen auf einen Messias von Wassermann aufgegriffen und im Haupt- und Nachfolgeroman erneut die Problematik des jüdischen Messianismus thematisiert. Die Analyse zeigt, dass in dem mit zahlreichen Stereotypen versehenen Werk, das Bild des »falschen Messias« eine dominante Stellung einnimmt. Deshalb wird überwiegend das Motiv des »Verrates« durch die Darstellung des 
messianischen Glaubens dargestellt; zumal auch die Agathon-Handlung aus einem Vorspiel, Die Juden von Zirndorf und Die Geschichte der jungen Renate Fuchs besteht.

Im Werk Jakob Wassermanns wird historische Faktizität mit literarischer Fiktionalität verschmolzen, so dass beispielsweise die Figur Sabbatai Zewi im Vorspiel und in der Haupthandlung nicht pathologisch erscheint, wie es etwa beim SabbataiForscher Scholem der Fall ist. Ferner werden im Werk auch primär die messianischen Züge im Hinblick auf die Hoffnung und die anschließende Enttäuschung nach der Apostasie, die Judenfrage im Allgemeinen und das Motiv der »Heimat « sowie der »Passivität« in Anlehnung an den Ur-Zewi dargestellt und somit die Bilder der Judenfeindschaft vergegenwärtigt.

Wichtig im realen und historischen Sabbatai-Stoff ist exempli causa die Verwendung von einem Verkünder im Namen Zewis, der für den sabbatianischen Erfolg der Überzeugung wichtig ist. Das hat Wassermann nämlich übernommen, und durch den Akt der Manipulation romanintern Spannung aufgebaut. Der Höhepunkt des Romans wird dann mit dem Schock und der Enttäuschung der jüdischen Gemeinschaft nach der Apostasie Zewis in der Handlung dargestellt. Der »Verräter « der Religion wird mit dem stereotypen Bild von Judas dargestellt, da Zewi einen Verrat an der eigenen Gemeinschaft ausübt.

Das Auftreten des Verkünders und Predigers, Zacharias Naar, zeigt, wie explizit die Beschuldigung an Juden in Worte gefasst werden. Dabei treten die Bilder der Judenfeindschaft auf, wie etwa das Bild des »Gottesmörders«, »Brunnenvergifters«, »Ritualmörders «, »Blutsaugers« und »Vampirs«.

\footnotetext{
Er (=Zacharias Naar) sprach zuerst in hastig hingeworfenen Worten von der Niedrigkeit und Erbärmlichkeit des jüdischen Volkes; von der Unterdrückung, die es erlitten, und von der Zerstreuung in alle Teile der Welt. [...] Er rief den alten Gott der Juden an, der Verheißung auf Verheißung gehäuft und die Armut über sein erwähltes Volk geschüttet habe und die Qualen der Heimsuchung, ärger als zur Zeit der ägyptischen Plagen. [...] Der Redner fuhr fort: ,Der Zorn des Herrn ist entbrannt wider sein Volk, und er streckt seine Hand wider sie aus und er schlägt es, so daß die Berge erzittern und ihre Leichen wie Kehricht mitten auf den Straßen liegen. Haben sie uns beschuldet: ,Ihr vergiftet unsere Brunnen'? Haben sie nicht unsere Brüder hingeschlachtet zu Tausenden? Haben sie nicht geschrien: ,Ihr nehmt das Blut unserer Kinder zum Opfer beim Passahfeste'? ,Ihr nehmt das Blut und braucht es für eure schwangeren Weiber"? Haben sie und nicht ausgewiesen aus ihren Städten und unsere Häuser verbrannt? Und unsere Güter geraubt? Müssen wir nicht vogelfrei dahinwandern, und viele finden keine Hütte wie Kain, der seinen Bruder erschlug? Haben sie uns nicht aus Rad geflochten und den Henkern im Land preisgegeben wie krankes Vieh? Nicht unsere Kinder verbrannt, nicht unsere Weiber geschändet und als die Pest kam, nicht schlimmer unter uns gewütet denn die Pest? Bei alledem hat sich der Zorn des Herrn nicht gewandt.'(Wassermann 1995: 14)
}

Der Prediger geht dabei immer wieder auf das jüdische Schicksal ein und stellt das Leben in Niedrigkeit, Erbärmlichkeit und Unterdrückung, das Exil, die Qualen der Heimsuchung, die Verbrennung von Städten und Dörfern, die Ermordung zahlreicher Juden, den Raub und die Schändung von Frauen und Kindern dar. 
Die Judenhoffnung, die im Vorspiel mit höchster Enttäuschung ausgeht, wird dann im Roman erneut ins Zentrum gerückt und spielt im jüdischen Leben eine wichtige Rolle. Die Figuren im Roman sind nämlich die Nachfahren der Familie Maier im Vorspiel. Die vom Autor vorgenommene fiktive Umstrukturierung über die Vorfahren ist auch von Bedeutung, denn es entspricht nicht den realhistorischen Gegebenheiten. Demnach ist der Protagonist im Zirndorf-Roman einer der Urenkel von der Tochter Rachel und Studiosus, einem Christen. Agathon tritt demzufolge nicht als ein „Volljude“" (Sicroff 1960: 32) auf.

Zudem werden der Figur Agathon im Roman messianische Züge und Eigenschaften von Jakob Wassermann zugeschrieben. Doch die Arbeit zeigt, dass Agathon weniger die Rolle eines Messias, sondern vielmehr die des Offenbarers - wie etwa der Apostel Johannes - einnehmen kann.

Der Protagonist neigt in dem Roman allerdings nicht zur Dekadenz und somit zum charakterlichen Verfall, sondern eher zum Guten und zur Höherentwicklung, denn er zeichnet eine positive charakterliche Entwicklung und wird deshalb von seiner Umgebung als der Messias anerkannt. Die messianische Charakterzuschreibung macht den Protagonisten - der auch die einzige jüdische Hauptfigur in allen Werken von Wassermann ist - zu keinem Messias, weil er für den Tod einer weiteren Figur im Werk verantwortlich ist. Ferner entsprechen Verbrechen nicht den idealen Charaktereigenschaften eines Erlösers. Der Protagonist zeigt zwar einen Prozess vom individuellen Gesinnungswandlung, doch er stirbt am Ende des Fortsetzungsromans.

Agathons Offenbarung von einem weiteren Messias ist bedeutsamer, denn hierbei kann es sich nur um seinen Sohn Beatus handeln. Zur Etymologie des Begriffs »Beatus« ist zu sagen, dass er aus dem Lateinischen stammt und der »Selige« bzw. "Geheiligte« bedeutet. Hier offenbart sich die Wassermann'sche Vorstellung der symbolisch-messianischen Idee. Beatus verkörpert, wie dem Ende des Romans zu entnehmen ist, die Frucht der Liebe, und nur durch den Weg der gegenseitigen und bedingungslosen Zuneigung können Ängste und Vorurteile abgebaut werden. Mit Beatus dem Seligen wird das neue Geschlecht des Übermenschen im Sinne Wassermanns erblühen und jedes einzelne Individuum das Messianische im Inneren tragen, allein aufgrund der individuellen Selbstentscheidung handeln, Negatives absorbieren und innerlich vernichten. Er symbolisiert die »Frucht der Schöpfung« des neuen Geschlechts, so wie die verbotene Frucht vom Baum der Erkenntnis, die die Schöpfung des Diesseits darstellt, weil sie am Ende des Zirndorf-Romans nicht ausgereift ist.

\section{Der Wassermann'sche Übermensch}

Das Konzept vom Wassermann'schen Übermenschen lehnt sich stark an das von Nietzsche an, ${ }^{6}$ da sich dadurch für Wassermann ein gedanklicher Ausweg ergibt, weil er

\footnotetext{
${ }^{6}$ In der Forschung gehen vorrangig Jütten (2007) und Haberich (2013) (2015) (2017) auf die Wassermann'sche Konzeption vom Übermenschen durch den Einfluss von Nietzsche ein. Beide
} 
vom Europagedanken nichts hält ${ }^{7}$ und die deutsch-jüdische Symbiose trotz des eigenen Dualismus von Deutsch-Sein und Jude-Sein für ihn weiterhin als eine Illusion verbleibt. Darüber hinaus begegnet er der zionistischen Bewegung mit großer Distanz und schließt sich dem nicht an (vgl. Spiel 1984: 13). Das deutungsoffene Konzept von Nietzsche bietet nämlich den Lösungsweg für eine bessere Zukunft, die Wassermann als die »Heimat« bezeichnen könnte. Wassermann sieht m. a. W. die Aufklärung des 18. und 19. Jahrhunderts und die jüdische Aufklärung in ihrer Mission als unvollkommen an und fordert deshalb die Menschheit zur Höherentwicklung zum Übermenschen auf.

Der Übermensch in Form eines Erlösers in Person bzw. Messias - so wie es Jütten (vgl. 2007: 193, 197) und Haberich (vgl. 2013: 16-18) nennen - trifft an dieser Stelle auch nicht ganz zu, denn hier geht es primär darum, dass Wassermann anhand der Pointierung auf Stereotype und der Grundlage von Nietzsche eine Erlösung im Sinne der Loslösung von der Vergangenheit beabsichtigt und auf die von Furcht und Unwissenheit begründeten Vorurteile und Voreinstellungen, die ihre Ausdrucksform sogar in Form der Gewalt zeigen, aufmerksam macht: „Denn dass der Mensch erlöst werde von der Rache: das ist mir die Brücke zur höchsten Hoffnung“ (Colli 1999: 128). Die Befreiung von der Rache heißt auch sich von der Vergangenheit loszulösen:

Das Vergangne am Menschen zu erlösen und alles ,Es war' umzuschaffen, bis der Wille spricht: ,Aber so wollte ich es! So werde ich's wollen-' [...]

An euren Kindern sollt ihr gut machen, dass ihr eurer Väter Kinder seid: alles Vergangene sollt ihr so erlösen! (Colli 1999: 249ff.)

Jütten ist soweit zuzustimmen, dass man sich beim (Über-)Menschen auf das Göttliche fokussieren soll, um fremdfeindliche Bilder abzubauen (2007: 184). Ferner kann die bisherige Feststellung von Jütten und Haberich erweitert werden, da die Entwicklung des eigenen Ichs von innen heraus zum Übermenschen durch alle Wassermann'schen Protagonisten illustriert werden. Vor allem seine Hauptfiguren in den Romanen nach dem Ersten Weltkrieg zeigen dies umso dominanter, da das Elend des Krieges, der steigende Antisemitismus und der Mord an Walther Rathenau (vgl. Kraft 2008: 173) Wassermann seelisch tief getroffen $\mathrm{zu}$ haben scheinen und er sich deshalb vom

verwenden den Begriff in Anbetracht auf Wassermanns Protagonist Agathon. Mit ihm wolle Wassermann nämlich den Übermenschen darstellen. Das Konzept des Übermenschen spielt darüber hinaus auch im Nationalsozialismus eine wichtige Rolle, wo es den eigenen Werten und Ideologien entsprechend instrumentalisiert wurde. Dies entspricht allerdings weder den Vorstellungen Nietzsches, noch sind sie für die vorliegende Arbeit relevant. Hier dienen vor allem die Arbeiten von Elisabeth Jütten und Max Haberich in Bezug auf den Übermenschen als Vorlage. Alle anderen Deutungsversuche, vor allem die des Nationalsozialismus', entsprechen nicht Sinn und Zweck der Analyse.

7 In Selbstbetrachtungen zeigen sich Wassermanns Gedanken über Europa am deutlichsten, denn er bezeichnet ihn als eine „Fiktion“. Der steigende Antisemitismus, den er tief spüren musste, und die Antipathie gegenüber dem modernen assimilierten Juden sorgen für die Distanzierung und Abkehr vom Europagedanken: „Was vor allem, verstehst du unter einem europäischen Menschen, unter europäischen Geist? Es ist eine unhaltbar gewordene Fiktion. Man darf es ja heute kaum wagen, als Europäer zu denken, und als Europäer zu dichten, gilt beinahe als Verrat. Europa hat sich selbst aufgegeben, wie kann es uns tragen? (s. Wassermann, Jakob (1984a): Selbstbetrachtungen. In: Rodewald, Dirk (Hrsg.) (1984): Jakob Wassermann. Deutscher und Jude. Reden und Schriften 1904-1933. Heidelberg: Lambert Scheider. S. 221). 
jüdischen Sachgehalt in seinen Romanen distanziert und eher auf das Problem der Gerechtigkeit seinen Schwerpunkt setzt.

In Also sprach Zarathustra führt die Erzählinstanz den Übermenschen ein: „Ich lehre euch den Übermenschen. Der Mensch ist Etwas, das überwinden werden soll“" (Colli 1999: 14). Dafür verwendet Nietzsche unter anderem die darwinistische These der Evolutionstheorie und setzt am Entwicklungsprozess an. Wenn der Affe demnach eine Entfaltung zum Menschen darstellen kann, so ist der Mensch in der Lage, sich zum Übermenschen weiterzuentwickeln, der letzten Endes der ganze Sinn der Erde ist:

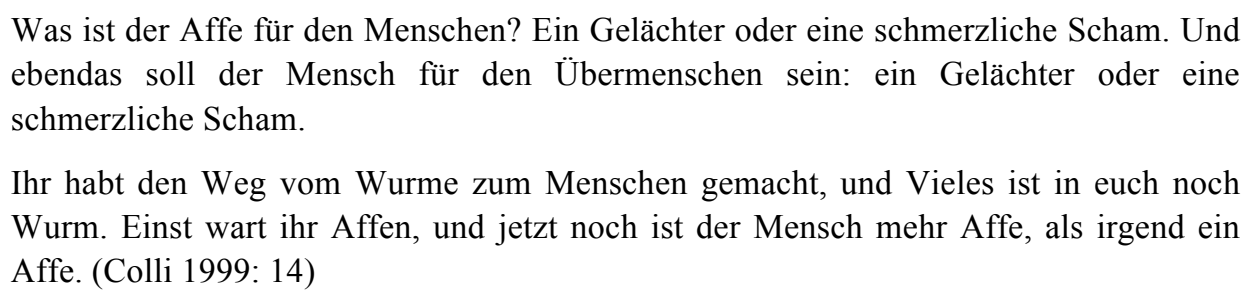

Die Ideenträger wie Christian Wahnschaffe, Arnold Ansorge, Etzel Andergast und Agathon Geyer zeigen primär die Eigenschaften des Übermenschen. Sie lehren zuerst das eigene Ich zu ehren, zu schätzen und zu lieben, sodass man auch den Nächsten lieben kann. Die Menschen schätzen sich nämlich nicht, sie „halte[n] es mit [sich] selber nicht aus und lieb[en] [sich] nicht genug. [...] [S]o muss [man] aus [ihnen] selber [den] Freund und [das] überwallende Herz schaffen“ (Colli 1999: 77). Der Primat der Liebe steht dementsprechend im Mittelpunkt, um Vorurteile überhaupt abzubauen.

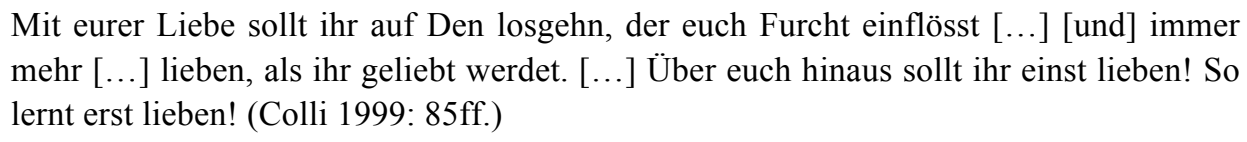

Ein Appell von Nietzsche geht also an die Menschheit, denn Menschen sollen sich einander bedingungslos und vorurteilsfrei lieben, um für Gerechtigkeit zu sorgen. Dass beispielsweise Arnold die Menschen liebt, und sich für den Tod entscheidet, um Gerechtigkeit zu schaffen, und somit für die ganze Menschheit sühnt, hebt die Wichtigkeit und Notwendigkeit der Liebe und Gerechtigkeit hervor:

\footnotetext{
Gerechtigkeit ohne Liebe ist nicht denkbar. Dass es Gerechtigkeit ohne Liebe wollte, in Gerechtigkeit leben wollte ohne Liebe, daran ist das Judentum gescheitert. Ein gerechtseiender Gott ist ein Gott ohne Liebe. [...] Ich sage also: tretet aus der Erstarrung heraus; aus der lieblosen Gerechtigkeit heraus zur gerecht werdenden Liebe. (Wassermann 1984a: 264)
}

Aus dem deutungsoffenen Konzept des Übermenschen im Sinne Nietzsches, dem Primat der Liebe und der Auffassung von Gerechtigkeit und dem Messianismus kann man Wassermanns Grundposition zum Judentum ableiten, denn sie ist „Ausweis einer kritischen Solidarisierung, sie soll das messianisch-futuristische Element, das so oft fehlgeleitet ist, aktivieren und vom Jüdisch-Partikularen, Religiös-Transzendenten ins Menschlich- Universale, Weltimmanente ausweiten“" (Horch 1987: 138). Der diesseitsbejahende und universal-humane Übermensch ist das neue Produkt, die Wunschprojektion Wassermanns. Der überhumane Mensch aus der Wassermann'schen Symbiose von Deutsch- und Judentum soll nämlich alle negativen Elemente 
absorbieren, und mit Liebe für Recht und Gerechtigkeit im gesellschaftlichen Leben sorgen. ${ }^{8}$

Ontologisch wird die Gerechtigkeit als Liebe verstanden, die Empathie voraussetzt. Erst dem Einfühlungsvermögen gelingt das wahre Seelenportrait des Nächsten, welches nicht nur Gegenwärtiges erfasst, sondern auch das (gute) Potenzial, die wahrhafte Essenz des Menschen. In diesem Punkt verbinden sich bei Wassermann künstlerischer Anspruch und Gerechtigkeitsbemühen. Denn seine Zielsetzung, die [Ideenträger] zum ,Bild` zu erheben, ist verknüpft mit einem persönlichen (Jütten 2007: 220) ,Gleichgewichtsstreben [...], dessen höhere Form das Verlangen nach Gerechtigkeit ist. (Wassermann 1984a: 181) ,Chaos durch die Form zu bannen, auf nichts anderes kommt es an, für das Individuum, für die Nation, für die Menschheit‘. (Wassermann 1984a: 180)

Die Worte Wassermanns verdeutlichen in ganzer Klarheit, dass judenfeindliche Bilder mit Liebe und Gerechtigkeit und darüber hinaus, mit Empathie zu beseitigen sind. Um dieses Gefühl der Empathie bei seiner Leserschaft zu vermitteln, stellt Wassermann die Bilder in seinen Werken vor, die seit der Entstehung des Kalenders tradiert werden. Die Aussagen Wassermanns haben allerdings einen allgemeingültigen Charakter wie der kategorische Imperativ von Kant. Ein Appell an die Menschheit ist vorzufinden, denn man soll nicht zu Unrecht urteilen. Die hier erwähnte Liebe ist als die Bedingung des

\footnotetext{
${ }^{8}$ In dieser Hinsicht kann auch Adornos Konzept hineinbezogen werden, denn er führt sein Prinzip der Erziehung »nach Auschwitz« vor, so dass man stets an den Zivilisationsbruch erinnern soll, da er die „Zerstörung der Erinnerungen“ als einen Betrug gegen die gefallenen Opfer sieht. In seiner demokratischen Pädagogik ist zwar nicht eindeutig zu erkennen, wie die Erziehung stattfinden soll, allerdings fügt er an, dass durch die Hinwendung ,zum Subjekt“ in Form der Stärkung des aufgeklärten Selbstbewusstseins, die grausamen Taten der Menschen aufgedeckt und ein allgemeines Bewusstsein erweckt werden soll, damit die Geschichte in ihrer Grausamkeit sich nicht wiederhole: „Man muss die Mechanismen erkennen, die die Menschen so machen, dass sie solcher Taten fähig werden, muss ihnen selbst diese Mechanismen aufzeigen und zu verhindern trachten, dass sie abermals so werden, indem man ein allgemeines Bewusstsein solcher Mechanismen erweckt"“(Adorno 1970, S. 95.).

Wie die Versöhnung des jüdischen Kollektivs mit seinem Täter ermöglicht werden soll, bleibt zwar bei Adornos Erziehung offen, doch Wassermann plädiert in dieser Hinsicht auf das Primat der Liebe und eine vorurteilsfreie Grundhaltung gegenüber dem Anderen. Die Versöhnung der Juden mit seinem Täter kann allerdings nach dem Holocaust nur erschwert möglich sein, da verschiedene schwerwiegende Paradigmen eine Rolle spielen. Eine Versöhnung wäre zwar für ein zukunftsorientiertes Miteinander notwendig, doch Vieles spricht dagegen. Erstens ist es die Tatsache, dass zeitgenössische Juden auf der einen Seite die Wiedergutmachungsbestrebungen anerkennen und respektieren, doch auf der anderen Seite den Tätern und Verantwortlichen nicht vergeben können. Das Recht der Vergebung liegt nämlich nicht primär in den Händen zeitgenössischer Juden, sondern vielmehr in den Händen der gefallenen Opfer und den noch lebenden jüdischen Zeitzeugen. Die jüdische Gnade kann nämlich - falls sie erfolgen sollte - als eine Enttäuschung, Betrug und/oder Verrat wahrgenommen werden. Zweitens würde die Verzeihung auch bedeuten, dass Juden das historische Erbe des NS-Regimes anerkennen und akzeptieren, wobei es im Grunde genommen nicht ihr eigenes und nicht ihr Handeln ist. Das würde die Opfer des Zweiten Weltkrieges gemeinsam mit den Tätern dieselbe Erbschaft, das selbstverständlich mit der Frage der Schuld eng in Zusammenhang steht, zuschreiben. Drittens widerspricht es der menschlichen Natur seinem Verbrecher mit Liebe entgegenzukommen. Deshalb kann im Hinblick auf den Übermenschen nur die These von Adorno mit hineinbezogen werden, die zur Sensibilisierung der Gemeinschaft durch die „Wendung aufs Subjekt“ (Adorno 1970: 95) beiträgt. Es ist hier noch einmal festzuhalten, dass Wassermann den Holocaust nicht erlebt hat, da er kurz davor starb. Wichtig für uns ist allerdings, wie sein Schaffen die Erziehung, Pädagogik, Wahrnehmung und das Handeln nach Auschwitz beeinflusst. Schließlich ist noch zu erwähnen, dass das Konzept des Übermenschen für eine Loslösung von der Vergangenheit spricht, wobei im Adorno'ischem Sinne dies der umgekehrte Fall ist, denn man soll stets an Auschwitz, also an die Vergangenheit, erinnern.
} 
gegenseitigen Verstehens zu lesen: Nur dadurch würde man sich vor dem Anderen nicht mehr fürchten müssen.

Die Kraft der inneren Liebe kann also die Herzen reinwaschen und das »Recht auf Leben« ermöglichen, das über allen Religionen steht. Das „Blut vergiftet[e] [nämlich] die reinste Lehre [...] zu Wahn und Hass der Herzen“ (Colli 1999: 119).

\section{Abschließende Bemerkung}

Gegenstand der vorliegenden Untersuchung war die Darstellung des ausgeprägten Ressentiments gegenüber dem Judentum anhand ausgewählter Werke Jakob Wassermanns, der sich mit dem Thema literarisch intensiv auseinandergesetzt hat. In den analysierten Werken rückt auch die Judenfrage immer wieder in den Vordergrund. Die Bilder der Judenfeindschaft sind durch Werk und Wirkung Wassermanns einer größeren Leserschaft zugänglich gemacht worden. In den Werken des Autors kann der funktionalen Bedeutung der Protagonisten als Ideenträger Wassermann'scher Gedanken eine strategische Schlüsselfunktion zugewiesen werden. Im literarischen Schaffen Wassermanns sind auch der Einfluss der kabbalistischen Lehre, die jüdische Mystik wie auch die Auffassung von Gerechtigkeit von Bedeutung und stehen infolgedessen im Mittelpunkt der Betrachtung. Das Ziel der Untersuchung bestand auch in der Darstellung judenfeindlicher Bilder in ausgewählten Werken des deutsch-jüdischen Schriftstellers.

Um der Voreinstellung gegenüber dem Anderen und folglich der Absonderung entgegenzuwirken und eine Sensibilisierung seiner Leserschaft zu ermöglichen, hat Wassermann von den historisch tradierten stereotypen Bildern Gebrauch gemacht. Wie in der Einleitung bereits erwähnt, will er seinen Ideen Gehör verschaffen. Die Wunschprojektion einer Gemeinschaft aus hochentwickelten Menschen, mit anderen Worten das Konzept des Übermenschen, sieht er als den einzigen Ausweg und spricht das Gewissen der Menschen an, sich den humanitären Eigenschaften zu nähern, stereotype und negative Gefühle und Empfindungen, die auf Vorurteile und tradierte Bilder beruhen, abzubauen und aufzulösen. Dies kann nur mithilfe der Empathie zustande gebracht werden, natürlich unter der Bedingung, dass man sich dem Anderen nähert und mit dem Unbewussten auseinandersetzt. Das bewusste und gezielte Vorgehen gegen die Ausgrenzung kann die kognitiven Verankerungen der Vorurteile, Stereotype und antijüdischen Bilder aus der Welt schaffen. Deshalb stehen Begrifflichkeiten wie Gerechtigkeit, Liebe, Gutmütigkeit, Willensbereitschaft, Menschlichkeit und Humanität im Zentrum der Wassermann'schen Überlegungen. Wie er es selber auch immer wieder erwähnt, ist die Gerechtigkeit ,[o]ffenbar [...] eine Gewichtslage der Seele. [...] In allen Fällen ist es eine Frage von Maß und Gewicht, eine, mit der die innere Substanz des Menschen zu tun hat" (Wassermann 1984a: 215f.).

Die Auseinandersetzung mit dem Anderen und die danach intendierte Änderung und Besserung in der Gefühlslage der Menschen soll keine stereotypen Verhaltensmuster mehr aufzuzeigen und demnach von der inneren Substanz des 
Menschen heraus entstehen. Um diese Bewusstwerdung und -machung zu ermöglichen, führt der Autor seine Protagonisten als Ideenträger ein. Aus der Analyse ist herzuleiten, wie die Hauptfiguren Agathon, Arnold, Christian und Etzel als Ideenträger fungieren und die Vorstellungen und Ideen Wassermanns von Gerechtigkeit und Liebe präsentieren. Da das Thema der Gerechtigkeit nicht im Zentrum dieser Arbeit stand, galt es im generellen Sinne ausfindig zu machen, in welcher Relation sie mit den stereotypen Bildern der Judenfeindschaft und den jüdischen Personen in den jeweiligen Werken steht.

\section{Literaturverzeichnis}

\section{Primärliteratur}

Wassermann, Jakob (1984a): Selbstbetrachtungen. In: Rodewald, Dirk (Hrsg.) (1984): Jakob Wassermann. Deutscher und Jude. Reden und Schriften 1904-1933. Heidelberg: Lambert Scheider. 221.

Wassermann, Jakob (1984b): Mein Weg als Deutscher und Jude. In: Rodewald, Dirk (Hrsg.): Jakob Wassermann. Deutscher und Jude. Reden und Schriften 1904-1933. Heidelberg: Lambert Scheider. 35-131.

Wassermann, Jakob (1995): Die Juden von Zirndorf. Cadolzburg: Arz Vivendi.

Wassermann, Jakob (2016a): Christian Wahnschaffe. Roman in zwei Bänden, 4. Aufl. Berlin: Holzinger.

Wassermann, Jakob (2016b): Der Fall Maurizius. 4. Aufl. Berlin: Holzinger.

Wassermann, Jakob (2017a): Der Moloch. 4. Aufl. Berlin: Holzinger.

Wassermann, Jakob (2017b): Die Geschichte der jungen Renate Fuchs. Berlin: Holzinger.

\section{Sekundärliteratur}

Assmann, Aleida (1994): Tradition, Evolution, Erinnerung. Überlegungen zum Strukturwandel kultureller Überlieferung. In: Goodmann-Thau, Eveline und Schmied-Kowarzik, Wolfdietrich (Hrsg.) (1994): Messianismus zwischen Mythos und Macht. Jüdisches Denken in der europäischen Geistesgeschichte. Berlin: Akademie, 89-199.

Assmann, Aleida (1999): Erinnerungsräume, Formen und Wandlungen des kulturellen Gedächtnisses. München: Beck.

Assmann, Jan (2000): Das kulturelle Gedächtnis. Schrift, Erinnerung und politische Identität in frühen Hochkulturen. München: Beck.

Assmann, Jan (2017): Religion und kulturelles Gedächtnis. München: Beck.

Assmann, Jan \& Tonio, Hölscher (1988): Kultur und Gedächtnis. Frankfurt am Main: Suhrkamp.

Baleaunu, Avram Andrei (1999):: Fünftes Bild: Der »ewige Jude«: In: Schoeps, Julius Hans und Schlör, Joachim (Hrsg.): Bilder der Judenfeindschaft. Antisemitismus Vorurteile und Mythen. Augsburg: Bechtermünz Verlag, 96-102.

Bausinger, Hermann (1988): Stereotypie und Wirklichkeit. In: Jahrbuch Deutsch als Fremdsprache 14, 157-170. 
Benz, Wolfgang (Hrsg.) (2010): Handbuch des Antisemitismus. Judenfeindschaft in Geschichte und Gegenwart. Band 3: Begriffe, Theorien, Ideologien. Berlin: de Gruyter.

Bitterli, Urs (2004): Die 'Wilden' und die 'Zivilisierten': Grundzüge einer Geistes- und Kulturgeschichte der europäischen-überseeischen Begegnung. München: Beck.

Buber, Martin (1960): Jüdische Renaissance. In: Buber, Martin (1960): Die Jüdische Bewegung, Band 1. Auflage 2. Berlin: Jüdischer Verlag, 7-16.

Colli, Giorgio\& Montinari, Mazzino (Hrsg.) (1999): Friedrich Nietzsche: Sämtliche Werke. Kritische Studienausgabe in 14 Bänden. 4. Bd. Also sprach Zarathustra. München: DTV.

Delumeau, Jean (1985): Angst im Abendland. Die Geschichte kollektiver Ängste im Europa des 14. bis 18. Jahrhunderts. 2 Bände. Hrsg. v. Johannes Beck. u.a. und übersetzt von Monika Hübner, Gabriel Konder und Martina Roters-Burck. Hamburg: Rowohlt.

Erb, Rainer (1999): Drittes Bild: Der »Ritualmörder«: In: Schoeps, Julius Hans und Schlör, Joachim (Hrsg.) (1999): Bilder der Judenfeindschaft. Antisemitismus Vorurteile und Mythen. Augsburg: Bechtermünz Verlag, 74-79.

Feinberg-Jütte, Anat (1999): Siebtes Bild: »Shylock«: In: Schoeps, Julius Hans und Schlör, Joachim (Hrsg.) (1999): Bilder der Judenfeindschaft. Antisemitismus Vorurteile und Mythen. Augsburg: Bechtermünz Verlag, 119-126.

Grözinger, Karl-Eric (1999): Erstes Bild: Die »Gottesmörder«. In: Schoeps, Julius Hans und Schlör, Joachim (Hrsg.) (1999): Bilder der Judenfeindschaft. Antisemitismus Vorurteile und Mythen. Augsburg: Bechtermünz Verlag, 57-66.

Haberich, Max Matthias Walter (2013): Arthur Schnitzler and Jakob Wassermann: A Struggle of German-Jewish Identities. Unpublished Dissertation. University of Cambridge. https://www.repository.cam.ac.uk/handle/1810/245043. (Zugriff: 15.04.2020).

Haberich, Max Matthias Walter (2015): Friedrisch Nietzsche and Jakob Wassermann: Brother in Spirit? In: Nietzsche-Studien. Internationales Jahrbuch für die Nietzsche-Forschung. Vol. 44, Issue I, 500-515.

Haberich, Max Matthias Walter (2017): Arthur Schnitzler und Jakob Wassermann: Konflikte deutschjüdischer Identitäten. In: Lukas, Wolfgang und Scheffel, Michael (Hrsg.) (2017): Textschicksale. Das Werk Arthur Schnitzlers im Kontext der Moderne. Berlin: de Gruyter, 123-138.

Heid, Ludger (1999): Achtzehntes Bild: »Der Ostjude«: In: Schoeps, Julius Hans und Schlör, Joachim (Hrsg.) (1999): Bilder der Judenfeindschaft. Antisemitismus Vorurteile und Mythen. Augsburg: Bechtermünz Verlag, 241-251.

Hortzitz, Nicoline (1999): Die Sprache der Judenfeindschaft. In: Schoeps, Julius Hans und Schlör, Joachim (Hrsg.) (1999): Bilder der Judenfeindschaft. Antisemitismus Vorurteile und Mythen. Augsburg: Bechtermünz Verlag, 19-40.

Karlweis, Marta (1935): Jakob Wassermann, Bild, Kampf und Werk. Mit einem Geleitwort von Thomas Mann. Amsterdam: Querido.

Kraft, Thomas (2008): Jakob Wassermann. München: DTV.

Lea, Henry Charles (1911): Geschichte der spanischen Inquisition. 3 Bde. 1980. Neudruck der Ausgabe Leipzig: Scientia.

Lüsebrink, Hans-Jürgen (2012): Interkulturelle Kommunikation. Interaktion, Fremdwahrnehmung, Kulturtransfer. 3. Aktualisierte und erweiterte Auflage. Stuttgart/ Weimar: Metzler.

Poliakov, Léon (1989): Geschichte des Antisemitismus. Worms: Heintz. 
Quasthoff, Uta M. (1973): Soziales Vorurteil und Kommunikation - Eine sprachwissenschaftliche Analyse des Stereotypes. Ein interdisziplinärer Versuch im Bereich der Linguistik, Sozialwissenschaft und Psychologie. Frankfurt am Main: Athenäum Fischer.

Raphael, Freddy (1999): Sechstes Bild: »Der Wucherer«: In: Schoeps, Julius Hans und Schlör, Joachim (Hrsg.) (1999): Bilder der Judenfeindschaft. Antisemitismus Vorurteile und Mythen. Augsburg: Bechtermünz Verlag, 103-118.

Rodewald, Dirk (Hrsg.) (1984): Jakob Wassermann. Deutscher und Jude. Reden und Schriften 19041933. Heidelberg: Lambert Scheider.

Rohrbacher, Stefan (1990): Eine Kreuztagung des 16. Jahrhunderts und ihr ikonographischer Kontext. In: Neusser Jahrbuch für Kunst, Kulturgeschichte und Heimatkunde. 35. Jg., 15-24.

Rohrbacher, Stefan \& Schmidt, Michael (1991): Judenbilder, Kulturgeschichte antijüdischer Mythen und antisemitischer Vorurteile. Hamburg: Rowohlt.

Schoeps, Julius H. (1986): Über Juden und Deutsche. Stuttgart: Burg.

Schoeps, Julius H. \& Schlör, Joachim (Hrsg.) (1999): Bilder der Judenfeindschaft. Antisemitismus, Vorurteile und Mythen. Augsburg: Bechtermünz.

Shaked, Gershon (1986): Der Fall Wassermann. In: Ders. (1986): Die Macht der Identität. Essays über jüdische Schriftsteller. Übersetzt von Ulrike Berger, Matthias Morgenstern und Eve Strauss. Königstein am Taunus: Athenäum. 95-114.

Spiel, Hilde (1984): Geleitwort. In: Rodewald, Dirk (Hrsg.) (1984): Jakob Wassermann. Deutscher und Jude. Reden und Schriften 1904-1933. Heidelberg: Lambert Scheider. 9-13.

Stölting, Erhard (1999): Sechzehntes Bild: »Der Verräter«. In: Schoeps, Julius Hans und Schlör, Joachim (Hrsg.) (1999): Bilder der Judenfeindschaft. Antisemitismus Vorurteile und Mythen. Augsburg: Bechtermünz Verlag. 218-228.

Wassermann-Speyer, Julie (1923): Jakob Wassermann und sein Werk. Wien und Leipzig: Deutschösterreichischer Verlag. 